\title{
Análise de estimabilidade de um modelo fenomenológico do processo de copolimerização do etileno em solução
}

\author{
I. N. Bessa ${ }^{1}$, K. V. Pontes ${ }^{1}$ \\ ${ }^{1}$ PEI - Programa de Pós-graduação em Engenharia Industrial - Universidade Federal da \\ Bahia \\ Email para contato: karenpontes@ufba.br
}

\begin{abstract}
RESUMO - Os modelos fenomenológicos para a polimerização normalmente apresentam dezenas de parâmetros a serem estimados, alguns inclusive correlacionados entre si. Além disto, há uma escassez de informações na literatura, o que dificulta a sua estimação direta. Para superar estas dificuldades, diversos métodos são propostos na literatura, dentre eles o tratamento prévio dos parâmetros através da análise de estimabilidade. O objetivo deste trabalho é aplicar a técnica de estimabilidade e ranqueamento baseada na ortogonalização para selecionar os parâmetros mais significativos do modelo fenomenológico da copolimerização do eteno com 1-buteno para posterior estimação a partir de dados dinâmicos fornecidos pela indústria. O modelo apresenta um total de 69 parâmetros, os quais foram reduzidos para 24 após o emprego da análise. A seleção dos parâmetros mais significativos simplificou o problema de otimização, de forma a se obter menores desvios entre a predição do modelo e os valores medidos. Além disso, a análise dos auto-valores da matriz Hessiana, proposta neste trabalho, indicou que o espaço formado pelos parâmetros selecionados apresenta um mínimo global. Este ponto mínimo foi encontrado na etapa da estimação. A análise de estimabilidade e consequente estimação permitiu a validação do modelo a partir das condições operacionais da planta.
\end{abstract}

\section{INTRODUÇÃO}

Na modelagem de processos complexos, como a polimerização, por exemplo, a estimação de parâmetros torna-se desafiadora. Os modelos fenomenológicos desenvolvidos para estes casos normalmente apresentam dezenas de parâmetros a serem estimados, alguns inclusive correlacionados entre si. Além disto, há uma escassez de informações na literatura, o que dificulta sua estimação direta. Para superar tais dificuldades, diversos métodos são propostos na literatura (Kravaris et al., 2013), dentre eles o tratamento prévio dos parâmetros através da análise de estimabilidade e ranqueamento baseada na ortogonalização.

A análise de estimabilidade já foi anteriormente empregada em diversos trabalhos (Benyahia et al., 2013; Jayasankar et al., 2009; Quaiser e Mönnigmann, 2009), porém a grande maioria destes tem o foco na estimação de parâmetros de modelos desenvolvidos a partir de uma planta piloto. Yao et al. (2003), por exemplo, analisam a estimabilidade de um modelo fenomenológico para a copolimerização do etileno/buteno com catalisadores Ziegler-Natta em fase gasosa a partir de dados experimentais obtidos de um reator piloto. O modelo é composto por um total de 50 parâmetros cinéticos. A análise possibilitou uma redução para um número entre 22 a 27 parâmetros, dependendo do critério de parada. 
Os autores mediram em linha algumas propriedades do polímero, como a fração molar de copolímero incorporada e o peso molecular. Na indústria, entretanto, tais análises nem sempre estão disponíveis e, quando estão, apresentam um elevado tempo morto de medição. Além disso, em escala laboratorial é possível planejar os experimentos a fim de obter dados para a estimação, enquanto que, em escala industrial, a estimação deve ser realizada através de dados históricos do processo. Desta forma, a estimação de parâmetros com dados de processo apresenta desafios e exige uma cuidadosa etapa preliminar de análise, justificando o emprego prévio da análise de estimabilidade para estes casos. Poucos trabalhos dão foco à questão da modelagem voltada para a aplicação na indústria. Dentre os trabalhos que utilizam dados industriais para a estimação de parâmetros, como Embiruçu et al. (2008) e Pontes et al. (2010), a análise de sensibilidade não foi realizada.

Quando se aborda modelos complexos, com um grande número de parâmetros é normalmente impossível estimar todo o conjunto de parâmetros devido às correlações entre eles e à quantidade limitada de dados disponíveis. Desta forma, tais análises devem ser realizadas antes da etapa de estimação, visando simplificá-la e consequentemente obter resultados mais precisos e de forma mais eficiente.

Segundo Quaiser e Mönnigmann (2009), os métodos de análise de estimabilidade buscam verificar se é possível determinar os parâmetros de um modelo a partir dos dados de entrada e saída disponíveis. Os autores classificam as seguintes técnicas de estimabilidade como as mais simples e eficazes: análise de componentes principais (Degenring et al., 2004; TURANYI, 1990); método do auto-valor (Quaiser e Mönnigmann, 2009); método da correlação (Jacquez e Arbor, 1985); método baseado na ortogonalização (Yao et al., 2003). A análise de estimabilidade baseada na ortogonalização foi desenvolvido por Yao et al. (2003) para substituir o método do auto-valor, devido ao seu elevado tempo de processamento. Existem duas abordagens para a análise de estimabilidade: estrutural e prática. A primeira baseia-se na estrutura do modelo, não necessita de dados experimentais para realizar a análise e depende da estimativa inicial dos parâmetros, enquanto a outra baseia-se em medidas experimentais.

A análise de estimabilidade pela ortogonalização apresenta a vantagem de considerar possíveis correlações e dependência linear entre parâmetros. Isto é importante pois as correlações podem inviabilizar ou mascarar os resultados da estimação dos parâmetros. Além disto, a abordagem estrutural para realizar a análise elimina a necessidade de dados experimentais.

Trabalhos anteriores concluíram que, para os casos apresentados, a estimabilidade baseada na ortogonalização conduz a resultados superiores quando comparada com outras técnicas (Benyahia et al., 2013; Quaiser e Mönnigmann, 2009; Yao et al., 2003). Portanto, o atual trabalho propõe utilizar o método da ortogonalização (Yao et al., 2003) para realizar a análise de estimabilidade dos parâmetros para o modelo da copolimerização do eteno, em escala industrial, com consequente estimação dos parâmetros. Além disto, neste trabalho, uma análise dos auto-valores da matriz Hessiana dos parâmetros ranqueados é proposta, de forma a avaliar o espaço formado pelos parâmetros ranqueados. Os auto-valores permitem indicar a existência de um mínimo global para o problema de otimização. Assim o método aqui utilizado será referido como Análise de Estimabilidade e Ranqueamento Baseada na Ortogonalização com Avaliação dos Auto-valores da Matriz 
Hessiana (AEROA).

A seção seguinte descreve o processo estudado. Em seguida o método de análise de estimabilidade baseado na ortogonalização é apresentado. A formulação do problema de estimação de parâmetros é detalhada. Os resultados são apresentados e discutidos. Este artigo se encerra com as conclusões.

\section{DESCRIÇÃO DO PROCESSO}

O caso de estudo configura-se na produção do polietileno linear de baixa densidade, através da copolimerização do etileno em solução de ciclohexano com catalisadores de Ziegler-Natta, conforme ilustra a Figura 1. O sistema é composto por dois reatores tubulares de fluxo pistonado (Plug Flow Reactor) e um CSTR (Continuous Stirred-Tank Reactor) não ideal, em operação adiabática. A alimentação é composta por uma mistura de eteno (monômero), co-monômero, hidrogênio (agente de transferência de cadeia), catalizadores (CAT), co-catalizador (CO-CAT) e ciclohexano (solvente).

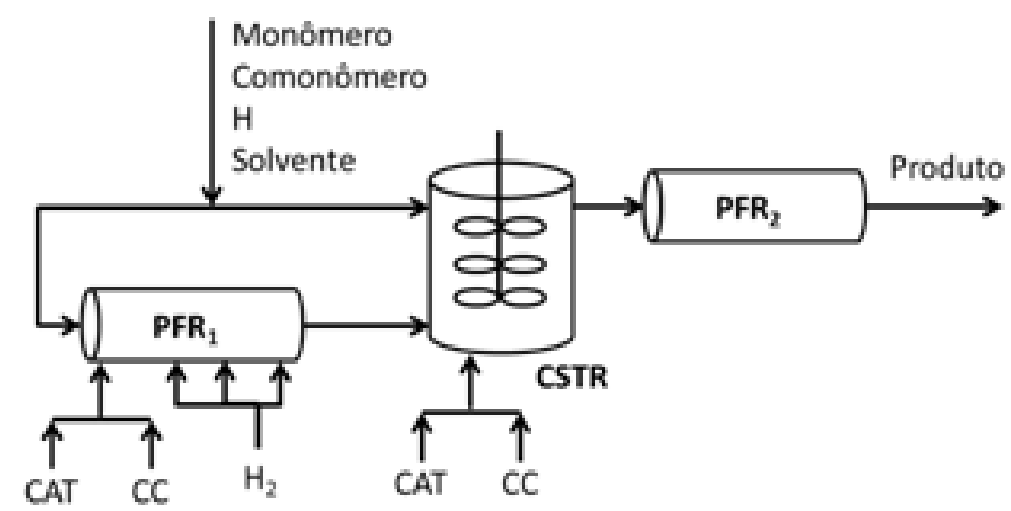

Figura 1 - Descrição do esquemática do processo. Fonte:Pontes et al. (2010).

Embiruçu et al. (2008) desenvolveu o modelo fenomenológico deste processo para o caso da homopolimerização do eteno. O modelo é composto por 36 parâmetros que foram estimados em Embiruçu et al. (2008) partir de dados coletados na planta industrial. Pontes et al. (2010) realizou a modelagem fenomenológica do processo para o caso da copolimerização do eteno. O modelo é composto por 69 parâmetros. Tendo em vista este elevado número de parâmetros e a escassez de dados de processo para estimá-los, os autores limitaram-se à validação qualitativa do modelo. Para que o mesmo possa ser aplicado na indústria, entretanto, é necessário que esteja validado quantitativamente.

\section{ORTOGONALIZAÇÃO}

O método AEROA é baseado na matriz de sensibilidade e na matriz Hessiana dos parâmetros ranqueados. Seja um processo dinâmico representado genericamente por:

$$
y=f(x(t), \theta, t)
$$


onde $y$ é o vetor de saídas do modelo, $x$, o vetor de condições operacionais do processo, $\theta$, os parâmetros do modelo e $t$, o tempo.

Os coeficientes da matriz de sensibilidade podem ser calculados através da derivada adimensional, conforme a equação:

$$
S_{i, p}=\left.\frac{\theta_{p}}{y_{i}} \frac{d y_{i}}{d \theta_{p}}\right|_{t=t_{n}} \quad i=1,2,3, \ldots, n_{y} \quad p=1,2,3, \ldots, n_{p} \quad n=1,2,3, \ldots, n_{t}
$$

onde $y_{i}$ é a resposta $i$ do modelo, $n_{y}$, o número de respostas avaliadas, $\theta_{p}$, o parâmetro do modelo, $n_{p}$, o número total de parâmetros, $t_{n}$, o instante de tempo em que a derivada é avaliada e $n_{t}$, o número de instantes de amostragem considerados. A normalização da matriz de sensibilidade através da derivada adimensional é recomendada por diversos trabalhos para manter todos os coeficientes da matriz de sensibilidade na mesma ordem de grandeza e na mesma dimensão, obtendo assim, uma melhor comparação do efeito de cada parâmetro (Benyahia et al., 2013; Lund e Foss, 2008; Yao et al., 2003). Desta forma, a matriz de sensibilidade terá dimensão $\left(n_{y} \cdot t_{n}\right) \times n_{p}$ e será representada por:

$$
S=\left[\begin{array}{ccccccc}
\left.S_{1,1}\right|_{t_{1}} & \left.S_{1,2}\right|_{t_{1}} & \left.S_{1,3}\right|_{t_{1}} & \cdots & \left.S_{1, \theta-2}\right|_{t_{1}} & \left.S_{1, \theta-1}\right|_{t_{1}} & \left.S_{1, \theta}\right|_{t_{1}} \\
\left.S_{2,1}\right|_{t_{1}} & \left.S_{2,2}\right|_{t_{1}} & \left.S_{2,3}\right|_{t_{1}} & \cdots & \left.S_{2, \theta-2}\right|_{t_{1}} & \left.S_{2, \theta-1}\right|_{t_{1}} & \left.S_{2, \theta}\right|_{t_{1}} \\
\vdots & \vdots & \vdots & \cdots & \vdots & \vdots & \vdots \\
\left.S_{y, 1}\right|_{t_{1}} & \left.S_{y, 2}\right|_{t_{1}} & \left.S_{y, 3}\right|_{t_{1}} & \cdots & \left.S_{y, \theta-2}\right|_{t_{1}} & \left.S_{y, \theta-1}\right|_{t_{1}} & \left.S_{y, \theta}\right|_{t_{1}} \\
\vdots & \vdots & \vdots & \ddots & \vdots & \vdots & \vdots \\
\left.S_{1,1}\right|_{t_{n}} & \left.S_{1,2}\right|_{t_{n}} & \left.S_{1,3}\right|_{t_{n}} & \cdots & \left.S_{1, \theta-2}\right|_{t_{n}} & \left.S_{1, \theta-1}\right|_{t_{n}} & \left.S_{1, \theta}\right|_{t_{n}} \\
\left.S_{2,1}\right|_{t n} & \left.S_{2,2}\right|_{t n} & \left.S_{2,3}\right|_{t n} & \cdots & \left.S_{2, \theta-2}\right|_{t n} & \left.S_{2, \theta-1}\right|_{t n} & \left.S_{2, \theta}\right|_{t n} \\
\vdots & \vdots & \vdots & \cdots & \vdots & \vdots & \vdots \\
\left.S_{y, 1}\right|_{t n} & \left.S_{y, 2}\right|_{t n} & \left.S_{y, 3}\right|_{t n} & \cdots & \left.S_{y, \theta-2}\right|_{t n} & \left.S_{y, \theta-1}\right|_{t n} & \left.S_{y, \theta}\right|_{t n}
\end{array}\right]
$$

Logo, cada coluna da matriz apresenta a influência de um determinado parâmetro, $\theta$, em relação às saídas, $y$, do modelo ao longo do tempo, $t$. O tempo final foi selecionado de forma que, para uma determinada condição de operação, o processo tenha tempo suficiente para alcançar o seu respectivo estado estacionário.

Uma vez definida a matriz de sensibilidade, torna-se necessário introduzir uma variável auxiliar, a magnitude de uma coluna, $M_{p}$, que é calculada por:

$$
M_{p}=S_{p}^{\top} \cdot S_{p}
$$

onde $S_{p}$ é uma determinada coluna da matriz de sensibilidade localizada na posição $p$.

O parâmetro mais estimável será aquele relacionado à coluna de maior magnitude, $p_{\text {max }}$. Selecionado o parâmetro mais estimável, a coluna referente a este parâmetro na matriz de sensibilidade deverá ser armazenada em uma matriz $S S$. A matriz de sensibilidade $S$ é então ortogonalizada em relação a $S S$, de modo a eliminar a correlação existente entre a coluna selecionada e as demais colunas da matriz. A ortogonalização é uma ferramenta algébrica que busca encontrar um vetor que seja linearmente independente e formado a partir de dois outros vetores linearmente dependentes, neste caso cada coluna de $S$ em relação a $S S$, conforme:

$$
S^{\prime}=S S \cdot\left(S S^{T} \cdot S S\right)^{-1} \cdot S S^{T} \cdot S
$$


onde $S^{\prime}$ é a matriz de sensibilidade ortogonalizada, cujas as colunas serão vetores linearmente independentes referência para a ortogonalização.

A projeção ortogonal de um vetor em relação a ele mesmo será o próprio vetor. Logo, a coluna de maior magnitude continuará com seu valor original na matriz após a ortogonalização. Para que a análise prossiga, é preciso obter uma matriz residual, $R$, que não inclua os valores da coluna anteriormente selecionada, através da equação:

$$
R=S^{\prime}-S
$$

A partir da matriz residual, as magnitudes são novamente calculadas, obtendo-se assim, o próximo parâmetro com maior influência na resposta do modelo. Os passos de ortogonalização e cálculo da matriz residual são então repetidos. A cada nova iteração uma coluna da matriz $S$ é adicionada à matriz $S S$. Ao fim do processo, está matriz representará a matriz de sensibilidade dos parâmetros selecionados. A ordem de seleção de cada parâmetro a partir da equação 4 definirá o rank destes, sendo que o primeiro selecionado será o de maior influência, e o ultimo o de menor. Este processo continuará até que a maior magnitude seja inferior ao critério de paragem, chamado valor de corte, $c$. Tal valor deverá ser definido para cada caso em estudo e estará diretamente relacionado com a convergência da análise. A garantia de que o espaço formado pelos parâmetros selecionados apresente um mínimo global e que não existem correlações é feita através da análise dos auto-valores da matriz Hessiana. A matriz Hesiana deverá ser obtida a partir da matriz de sensibilidade dos parâmetros ranqueados, $S S$. Se todos os auto-valores forem positivos, não existirá correlações entre os parâmetros e o espaço formado apresentará um mínimo global, mínimo que poderá ser encontrado na etapa de estimação.

A Figura 2 apresenta um algoritmo simplificado encontra-se uma representação esquemática do método aqui descrito: Análise de Sensibilidade Baseada na Ortogonalização com Avaliação dos Auto-valores da Matriz Hessiana.

\section{Estimação dos Parâmetros}

A estimação de parâmetros constitui uma das etapas do desenvolvimento de um modelo matemático, sendo fundamental para a validação e utilização do mesmo. Esta etapa consiste na solução de um problema de otimização para determinar os valores numéricos dos parâmetros em um determinado espaço de dados experimentais.

De forma a testar a eficiência do método aqui apresentado a estimação dos parâmetros foi realizada a partir dos dados industriais, coletados na planta. Os procedimentos para o tratamento dos dados, formulação da funções objetivo e da estimativa inicial foram seguidos de acordo a Embiruçu et al. (2008). A função objetivo pode ser representada conforme:

$$
f o b=\min \left(V^{\top} \cdot V\right)
$$

onde $V$ é o vetor de diferença entre os valores preditos pelo modelo e os valores coletadas na planta.

Foram estimados os 24 parâmetros indicados pela AEROA, os quais consistem em constantes cinéticas, viscosidades e propriedades na mistura. Duas variáveis de processo 
foram selecionadas para validar o modelo: a taxa de produção $(R A)$ e a temperatura na base do reator CSTR $\left(T_{b}\right)$. A produtividade representa a quantidade em massa de polímero produzido por unidade de tempo. Por sua vez a $T_{b}$ é um propriedade importante para o processo, uma vez que este é o ponto de alimentação de catalizador e a temperatura desta zona é responsável por ativar a reação catalítica sucessiva (SQP - Sequential Quadratic Programming).

O problema de otimização foi resolvido pela biblioteca dinâmica do Numerical Algorithms Group (NAG), que é baseada em na programação quadrática, SQP.

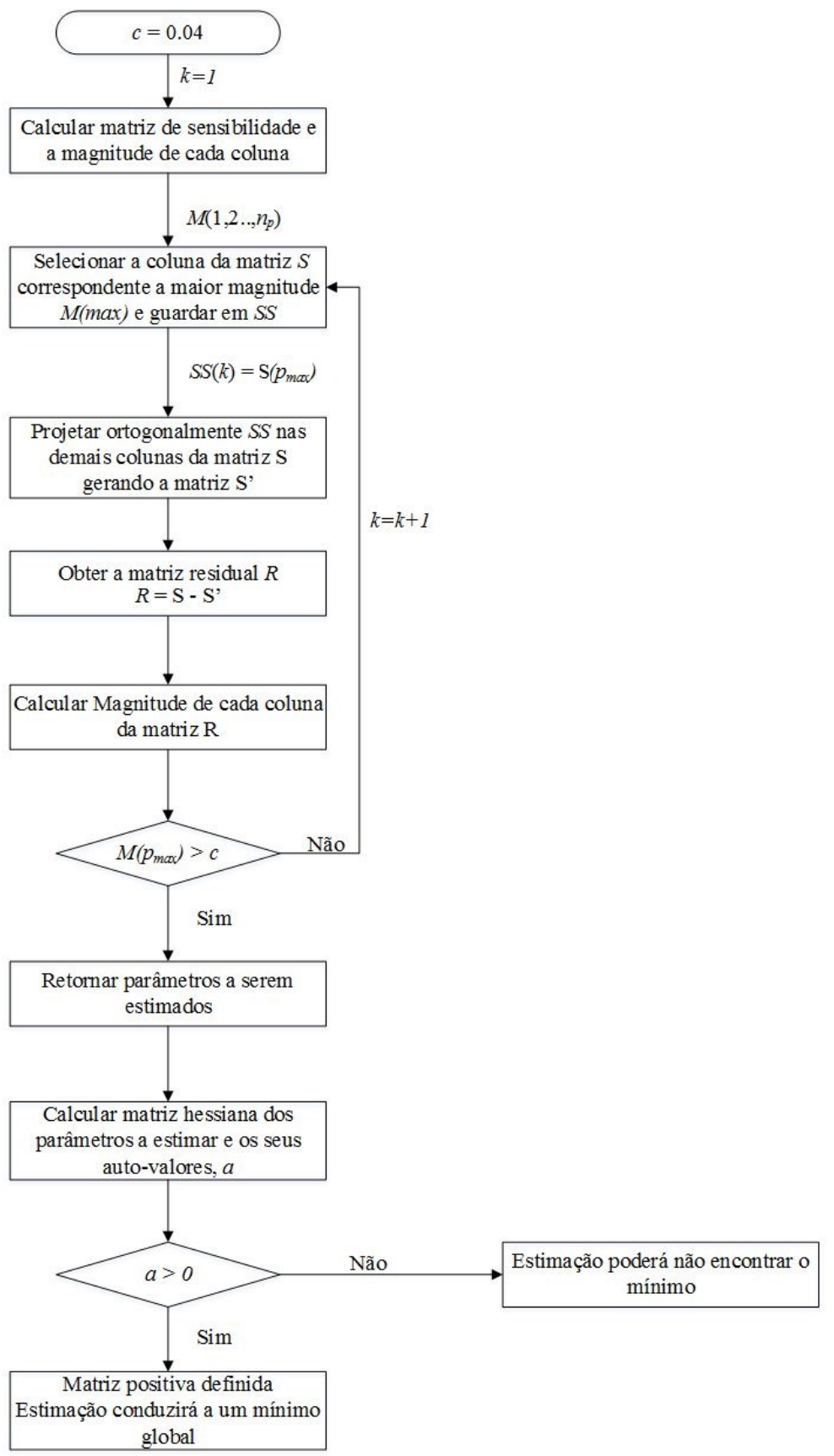

Figura 2 - Algorítimo do método AEROA. Fonte: Próprio Autor. 


\section{RESULTADOS}

Conforme mencionado anteriormente, o modelo fenomenológico (Pontes et al., 2010) apresenta 69 parâmetros. A matriz de sensibilidade do sistema possui uma dimensão de $(1200 \times 69)$, com $n_{y}=4, t_{n}=300$ e $n_{P}=69$. Ao aplicar a AEROA descrita na Figura 2, 24 parâmetros foram selecionados para estimação. Os parâmetros restantes permanecem constates, assumindo o valor de suas estimativas iniciais. A análise dos autovalores da matrz Hessiana formada pelos parâmetros ranqueados, dentre eles, constantes cinéticas, viscosidades e propriedades na mistura, demonstra que esta matriz é positiva definida, logo o espaço formado pelos parâmetros é estritamente convexo, de forma que a estimação destes parâmetros deverá conduzir a um ponto mínimo global. Na Figura 3 está representada a predição do modelo após a estimação com os dados experimentais. Todos os dados estão normalizados, por questões de confidencialidade. O percentual de variação do $R A$ foi de $-7 \%$ e de $6 \%$ para a $T_{B}$. Nota-se que o modelo é capaz de prever as saídas analisadas com desvios baixos em relação das dados do processo. Além disto, o modelo apresenta uma boa capacidade para a predição da dinâmica do processo.

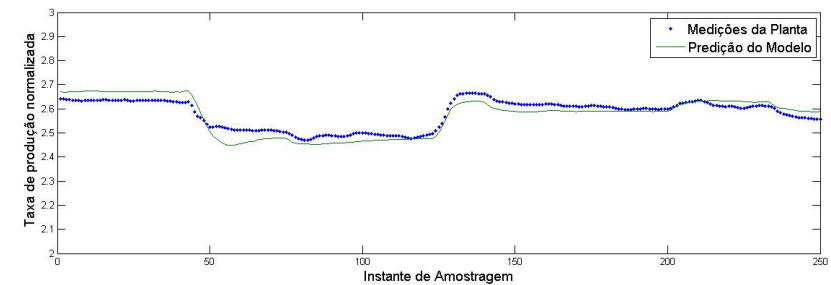

(a) Taxa de Produtividade Medida e Predita

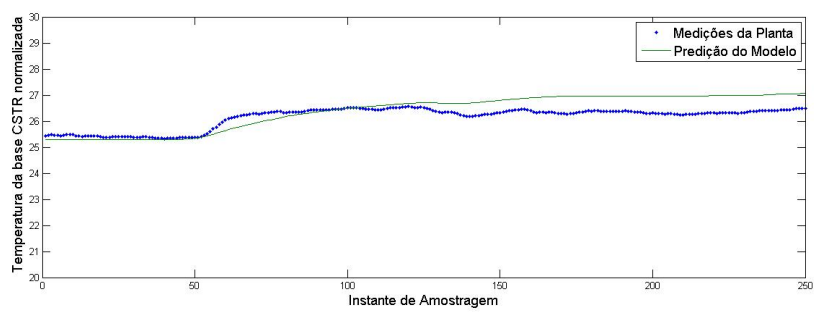

(b) Temperatura Medida e Predita

Figura 3 - Resultados obtidos pelo modelo estimado a partir dos resultados da AEROA.

\section{CONCLUSÔES}

Os resultados obtidos demonstram que a metodologia é capaz de analisar a estimabilidade os parâmetros do modelo de forma eficaz. A partir dos resultados deste trabalho é possível concluir que foi possível realizar com sucesso a análise de estimabilidade e ranqueamento dos parâmetros com base na ortogonalização e análise dos auto-valores da matriz Hessiana levando em consideração correlações; análise da influência de cada parâmetro nas resposta do modelo apresentado; redução significativa do número de parâmetros a ser estimados; análise do espaço formado pelos parâmetros ranqueados. 
Conclui-se que a metodologia aplicada foi eficiente no cumprimento dos objetivos propostos. A estimação detalhada dos parâmetros selecionado foi conduzida de uma forma simplificada. Os resultados da estimação dos parâmetros ranqueados demonstram que o modelo final apresenta uma boa capacidade de predição, com pequenos desvios dos dados industriais, inclusive prevendo a dinâmica do processo, o que é essencial para a aplicação industrial.

\section{REFERÊNCIAS}

Benyahia, B.; Latifi, M.; Fonteix, C.; Pla, F. Emulsion copolymerization of styrene and butyl acrylate in the presence of a chain transfer agent. Part 2: Parameters estimability and confidence regions. Chemical Engineering Science, 90, 110-118, 2013.

Degenring, D.; Froemel, C.; Dikta, G.; Takors, R. Sensitivity analysis for the reduction of complex metabolism models. Journal of Process Control, 14(7), 729-745, 2004.

Embiruçu, M.; Prata, D. M.; Lima, E. L.; Pinto, J. C. Continuous Soluble ZieglerNatta Ethylene Polymerizations in Reactor Trains, 2 - Estimation of Kinetic Parameters from Industrial Data. Macromolecular Reaction Engineering, 2(2), 142-160, 2008.

Jacquez, A.; Arbor, A. Numerical Parameter Identifiability and Estimability : Integrating Identifiability , Estimability , and Optimal Sampling Design. 227, 201-227, 1985.

JAYASANKAR, B. R.; Ben-Zvi, A.; HuAng, B. Identifiability and estimability study for a dynamic solid oxide fuel cell model. Computers $\& 3$ Chemical Engineering, 33(2), 484-492, 2009.

Kravaris, C.; Hahn, J.; ChU, Y. Advances and selected recent developments in state and parameter estimation. Computers \& Chemical Engineering, 51, 111-123, 2013.

Lund, B. F.; Foss, B. A. Parameter ranking by orthogonalization-Applied to nonlinear mechanistic models. Automatica, 44(1), 278-281, 2008.

Pontes, K.; Cavalcanti, M.; Filho, R. M.; Embiruçu, M. Modeling and simulation of ethylene and 1-butene copolymerization in solution with a Ziegler-Natta Catalyst. International Journal of . ., , 8, 2010.

QuAiser, T.; MÖnnigmann, M. Systematic identifiability testing for unambiguous mechanistic modeling-application to JAK-STAT, MAP kinase, and NF-kappaB signaling pathway models. BMC systems biology, 3, 50, 2009.

TURANYI, T. Reduction of large reaction mechanisms. New journal of chemistry, 14(11), 795-803, 1990.

Yao, K. Z.; Shaw, B. M.; Kou, B.; McAuley, K. B.; Bacon, D. W. Modeling Ethylene/Butene Copolymerization with Multisite Catalysts: Parameter Estimability and Experimental Design. Polymer Reaction Engineering, 11(3), 563-588, 2003. 